\title{
Alignment for the first precision measurements at Belle II
}

Tadeas Bilka ${ }^{1, *}$, Jesus Abudinen ${ }^{2}$, Karlheinz Georg Ackermann ${ }^{3}$, Karol Mateusz Adamczyk $^{4}$, Patrick Ahlburg ${ }^{5}$, Hiroaki Aihara ${ }^{6}$, Oscar Alonso ${ }^{7}$, Mohammed Albalawi $^{8}$, Ladislav Andricek ${ }^{9}$, Rachid Ayad ${ }^{8}$, Tariq Aziz ${ }^{10}$, Varghese Babu ${ }^{11}$, Szymon Grzegorz Bacher $^{4}$, Seema Bahinipati ${ }^{12}$, Giovanni Batignani ${ }^{13}$, Jerome Baudot ${ }^{14}$, Prafulla Kumar Behera $^{15}$, Stefano Bettarini ${ }^{13}$, Marca Boronat ${ }^{16}$, Andrzej Bozek $^{4}$, Nils Braun ${ }^{17}$, Florian Buchsteiner $^{18}$, Allen Caldwell ${ }^{3}$, Christian Camien ${ }^{11}$, Giulia Casarosa ${ }^{13}$, Daniel Cervenkov ${ }^{1}$, Vladimir Chekelian ${ }^{3}$, Yeqi Chen ${ }^{19}$, Kirill Chilikin ${ }^{43}$, Luigi Corona ${ }^{13}$, Thomas Rafael Czank ${ }^{20}$, Sanjeeda Bharati Das ${ }^{21}$, Nibedita Dash ${ }^{15}$, Gaetano de Marino ${ }^{13}$, Bruno Deschamps ${ }^{5}$, Angel Dieguez ${ }^{7}$, Jochen Dingfelder ${ }^{5}$, Zdeněk Doležal ${ }^{1}$, Giulio Dujany ${ }^{14}$, Daniel Esperante ${ }^{16}$, Francesco Forti ${ }^{13}$, Markus Fras $^{3}$, Ariane Frey ${ }^{22}$, Markus Friedl ${ }^{18}$, Juan Fuster ${ }^{16}$, Miroslav Gabriel $^{3}$, Karsten Gadow ${ }^{11}$, Eldar Ganiev ${ }^{2}$, Uwe Gebauer ${ }^{22}$, Thomas Gessler ${ }^{23}$, Georgios Giakoustidis $^{5}$, Luigi Li Gioi ${ }^{3}$, Benigno Gobbo ${ }^{2}$, Pablo Gomis López ${ }^{16}$, Daniel Greenwald ${ }^{24}$, Yinghui Guan ${ }^{25}$, Soumen Halder ${ }^{10}$, Koji Hara ${ }^{26}$, Oskar Hartbrich ${ }^{27}$, Sagar Hazra ${ }^{10}$, Martin Heck $^{17}$, Tomasz Hemperek ${ }^{5}$, Martin Hensel ${ }^{9}$, Takeo Higuchi ${ }^{20}$, Matthias Hoek ${ }^{28}$, Stefan Huber $^{24}$, Ryosuke Itoh $^{26}$, Christian Irmler ${ }^{18}$, Akimasa Ishikawa ${ }^{26}$, Hyebin Jeon ${ }^{29}$, Changwoo $\mathrm{Joo}^{20}$, Mateusz Kaleta ${ }^{4}$, Abdul Basith Kaliyar ${ }^{10}$, Jakub Kandra ${ }^{1}$, Kookhyun Kang ${ }^{29}$, Piotr Julian Kapusta ${ }^{4}$, Christian Kiesling ${ }^{3}$, Bartlomiej Kisielewski ${ }^{4}$, David Kittlinger ${ }^{3}$, Claus Kleinwort ${ }^{11}$, Daniel Klose ${ }^{9}$, Peter Kodyš ${ }^{2}$, Christian Koffmane ${ }^{9}$, T. Kohriky ${ }^{26}$, Tomoyuki Kono ${ }^{30}$, Igor Konorov ${ }^{24}$, Silvia Krivokuca ${ }^{9}$, Hans $\mathrm{Krüger}^{5}$, Thomas $\mathrm{Kuhr}^{31}$, Manish Kumar $^{21}$, Rajeev Kumar ${ }^{32}$, Peter Kvasnička ${ }^{1}$, Carlos Lacasta ${ }^{16}$, Chiara La Licata ${ }^{20}$, Kavita Lalwani $^{21}$, Livio Lanceri ${ }^{2}$, Jens Sören Lange ${ }^{23}$, Klemens Lautenbach ${ }^{23}$, Seungcheol Lee ${ }^{29}$, Ulrich Leis ${ }^{3}$, Philipp Leitl ${ }^{3}$, Dmytro Levit ${ }^{24}$, Chunhua $\mathrm{Li}^{33}, Y$. B. $\mathrm{Li}^{34}$, James Frederick Libby $^{15}$, Gerhard Liemann', Qingyuan Liu ${ }^{35}$, Zhen'An Liu ${ }^{36}$, Thomas Lück ${ }^{13,31}$, Florian Luetticke ${ }^{5}$, Lydia Macharski ${ }^{11}$, Souvik Maity ${ }^{12}$, Carlos Mariñas ${ }^{16}$, Sukant Narendra Mayekar ${ }^{10}$, Sara Mccarney ${ }^{3}$, Gagan Bihari Mohanty ${ }^{10}$, Johnny Alejandro Mora Grimaldo ${ }^{6}$, Tomoko Morii ${ }^{20}$, Hans-Günther Moser ${ }^{3}$, David Moya ${ }^{37}$, Felix Johannes Müller ${ }^{11}$, Felix Müller $^{3}$, Katsuro Nakamura ${ }^{26}$, Mikihiko $\mathrm{Nakao}^{26}$, Zbigniew Marian Natkaniec ${ }^{4}$, Carsten Niebuhr $^{11}$, Jelena Ninkovic ${ }^{9}$, Yoshiyuki Onuki ${ }^{6}$, Waclaw Ostrowicz ${ }^{4}$, Antonio Paladino ${ }^{13}$, Eugenio Paoloni ${ }^{13}$, Hwanbae Park ${ }^{29}$, SeokHee Park $^{38}$, Botho Paschen ${ }^{5}$, Stephan Martin Paul $^{24}$, Ivan Peric ${ }^{17}$, Frauke Poblotzki ${ }^{11}$, Andrei Rabusov ${ }^{24}$, K. K. Rao ${ }^{10}$, Simon Reiter ${ }^{23}$, Rainer Helmut Richter $^{9}$, Isabelle Ripp-Baudot ${ }^{14}$, Martin Ritter $^{31}$, Michael Ritzert ${ }^{39}$, Giuliana Rizzo ${ }^{13}$, Niharika Rout ${ }^{15}$, Debashis Sahoo $^{10}$, Javier Gonzalez Sanchez ${ }^{37}$, Luka Santelj $^{40}$, Nobuhiko Sato ${ }^{26}$, Bianca Scavino ${ }^{28}$, Gerhard Schaller ${ }^{9}$, Martina Schnecke ${ }^{9}$, Florian Schopper ${ }^{9}$, Harrison Schreeck ${ }^{22}$, Christoph Schwanda ${ }^{18}$, Benjamin Schwenker ${ }^{22}$, Reinhard Sedlmeyer ${ }^{9}$, Concettina Sfienti ${ }^{28}$, Frank Simon $^{3}$, Sebastian Skambraks ${ }^{9}$, Yuri Soloviev ${ }^{11}$, Björn Spruck ${ }^{28}$, Slavomira Stefková ${ }^{11}$, Reimer Stever ${ }^{11}$, Ulf Stolzenberg ${ }^{22}$, Soh Yamagata Suzuki $^{26}$, Maiko Takahashi ${ }^{11}$, Eva Tafelmayer $^{9}$, Shuji Tanaka ${ }^{26}$, Hikaru Tanigawa $^{6}$, Richard Thalmeier $^{18}$, Toru Tsuboyama ${ }^{26}$, Yuma Uematsu ${ }^{6}$, O. Verbycka ${ }^{4}$, Ivan Vila $^{37}$, Amparo Lopez Virto ${ }^{37}$, Lorenzo Vitale ${ }^{2}$, Sven $\operatorname{Vogt}^{3}$, Marcel $\operatorname{Vos}^{16}$, Kun $\mathrm{Wan}^{6}$, Boqun

\footnotetext{
*e-mail: bilka@ipnp.mff.cuni.cz
} 
Wang $^{3}$, Shun Watanuki ${ }^{41}$, James Webb ${ }^{42}$, Norbert Wermes ${ }^{5}$, Christian Wessel $^{5}$, Jarostaw Pawet Wiechczyński ${ }^{13}$, Philipp Wieduwilt ${ }^{22}$, Hendrik Windel $^{9}$, Satoru Yamada ${ }^{26}$, Hua $\mathrm{Ye}^{11}$, Hao Yin ${ }^{18}$, Laura Zani ${ }^{13}$, and Tingyu Zhang ${ }^{6}$ for the Belle II PXD, SVD, and Software collaborations.

${ }^{1}$ Charles University, Prague, Czech Republic

${ }^{2}$ INFN and Univ. Trieste, Italy

${ }^{3}$ Max Planck Institut fur Physik Muenchen, Germany

${ }^{4}$ Institute of Nuclear Physics PAN, Poland

${ }^{5}$ Univ. of Bonn, Germany

${ }^{6} \mathrm{U}$-Tokyo, Japan

${ }^{7}$ University of Barcelona, Spain

${ }^{8}$ Univ. of Tabuk, Saudi Arabia

${ }^{9}$ Semiconductor Laboratory of the Max Planck Society, Germany

${ }^{10}$ Tata Institute of Fundamental Research, India

${ }^{11}$ Deutsches Elektronen-Synchrotron(DESY), Germany

${ }^{12}$ Indian Institute of Technology Bhubaneswar, India

${ }^{13}$ INFN and Univ. Pisa, Italy

${ }^{14}$ Institut Pluridisciplinaire Hubert Curien (IPHC) Strasbourg, France

${ }^{15}$ Indian Institute of Technology Madras, India

${ }^{16}$ Instituto de Fisica Corpuscular(IFIC), Spain

${ }^{17}$ Karlsruhe Institute of Technology(KIT), Germany

${ }^{18}$ Institute of High Energy Physics, Austrian Academy of Sciences, Austria

${ }^{19}$ Univ. of Science and Technology of China(USTC), China

${ }^{20} \mathrm{Kavli}$ IPMU (WPI), the University of Tokyo, Japan

${ }^{21}$ Malaviya National Institute of Technology Jaipur, India

${ }^{22}$ Univ. of Goettingen, Germany

${ }^{23}$ Univ. of Giessen, Germany

${ }^{24}$ Technical Univ. of Munich(Technische Universitaet Muenchen), Germany

${ }^{25}$ University of Cincinnati, U.S.A.

${ }^{26}$ High Energy Accelerator Research Organization (KEK), Japan

${ }^{27}$ Univ. of Hawaii, U.S.A.

${ }^{28}$ Johannes Gutenberg Univ. of Mainz, Germany

${ }^{29}$ Kyungpook National Univ.(KNU), South Korea

${ }^{30}$ Kitasato University, Japan

${ }^{31}$ Ludwig Maximilians Univ. Muenchen(LMU), Germany

${ }^{32}$ Panjab Univ., India

${ }^{33}$ LiaoNing Normal University(LNNU), China

${ }^{34}$ Peking Univ.(PKU), China

${ }^{35}$ Fudan Univ., China

${ }^{36}$ Institute of High Energy Physics(IHEP), China

${ }^{37}$ Instituto de Fisica de Cantabria, Spain

${ }^{38}$ Yonsei Univ., South Korea

${ }^{39}$ Heidelberg University, Germany

${ }^{40}$ Univ. of Ljubljana, Slovenia

${ }^{41}$ Laboratoire de L'accelerateur Lineaire (LAL) Orsay, France

${ }^{42}$ Univ. of Melbourne, Australia

${ }^{43}$ Lebedev Physical Institute of the Russian Academy of Sciences, Russian Federation

Abstract. On March 25th 2019, the Belle II detector recorded the first collisions delivered by the SuperKEKB accelerator. This marked the beginning of the physics run with vertex detector.

The vertex detector was aligned initially with cosmic ray tracks without magnetic field simultaneously with the drift chamber. The alignment method is 
based on Millepede II and the General Broken Lines track model and includes also the muon system or primary vertex position alignment. To control weak modes, we employ sensitive validation tools and various track samples can be used as alignment input, from straight cosmic tracks to mass-constrained decays.

With increasing luminosity and experience, the alignment is approaching the target performance, crucial for the first physics analyses in the era of Super-BFactories. We will present the software framework for the detector calibration and alignment, the results from the first physics run and the prospects in view of the experience with the first data.

\section{Introduction}

The Belle II detector at the SuperKEKB $e^{+} e^{-}$accelerator (KEK, Tsukuba, Japan) [1] recorded its first collision data in the full configuration ${ }^{1}$ on March 25th 2019. It accumulated about $6.5 \mathrm{fb}^{-1}$ in the Spring run and in total about $10.57 \mathrm{fb}^{-1}$ in 2019 . Despite this being only a small fraction of the planned dataset of $50 \mathrm{ab}^{-1}$ at the $\Upsilon(4 S)$ resonance center-of-mass energy, it allows to study and optimize the performance of the detector and its operation in preparation for the record-breaking luminosity of the new generation of Super- $B$-Factory.

To ensure high performance, the detector has to be properly aligned and calibrated. Good alignment is of utmost importance namely for precision measurements of time-dependent $\mathrm{CP}$ violation - silicon sensors must be aligned significantly better than $10 \mu \mathrm{m}$ to prevent degradation of resolution. Reaching this goal involves not only alignment of the primary interaction region (IR) position and internal alignment of the vertex detector (VXD), but also relative alignment of the vertex detector with respect to the rest of the tracking system - the central drift chamber (CDC), as well as internal alignment of CDC itself. To reduce possible systematic effects, a single alignment procedure is in place for IR, VXD and CDC, which involves simultaneous internal and relative alignment of all the components. In addition, alignment of the $K_{L}$ and muon system (KLM) is integrated in the common procedure and several calibrations related to the CDC are considered or under development to be included as well.

Determination of many thousand of alignment parameters, which we briefly describe, is a considerable computing task, which we show to be achievable with common software tools/libraries and reasonable resources. Finally, results primarily from a reduced problem applied to the first cosmic and collision data are used to demonstrate the performance of the method.

\section{The Belle II detector and its alignment parameters}

The Belle II detector is a general purpose forward/backward asymmetric detector operating in $1.5 \mathrm{~T}$ solenoid magnetic field. The vertex detector is composed of two innermost layers of DEPFET pixel sensors (PXD) [2] and four layers of double-sided strip sensors (SVD) [3]. The sensors are organized in half-shells and ladders, which is reflected in the alignment hierarchy, see Fig. 1. The momentum of the particles is measured by the central drift chamber (CDC), surrounded by particle identification detectors and electromagnetic calorimeter. Outside of the solenoid is the $K_{L}$ and muon system (KLM) divided into barrel and two end-caps.

\footnotetext{
${ }^{1}$ The pixel detector differs from designed configuration - only first layer and four sensors from second layer are installed, because not all sensors were available at the time of the installation. A replacement of the pixel detector is foreseen in coming years, completing the second layer as originally designed.
} 


\section{Belle II Detector}

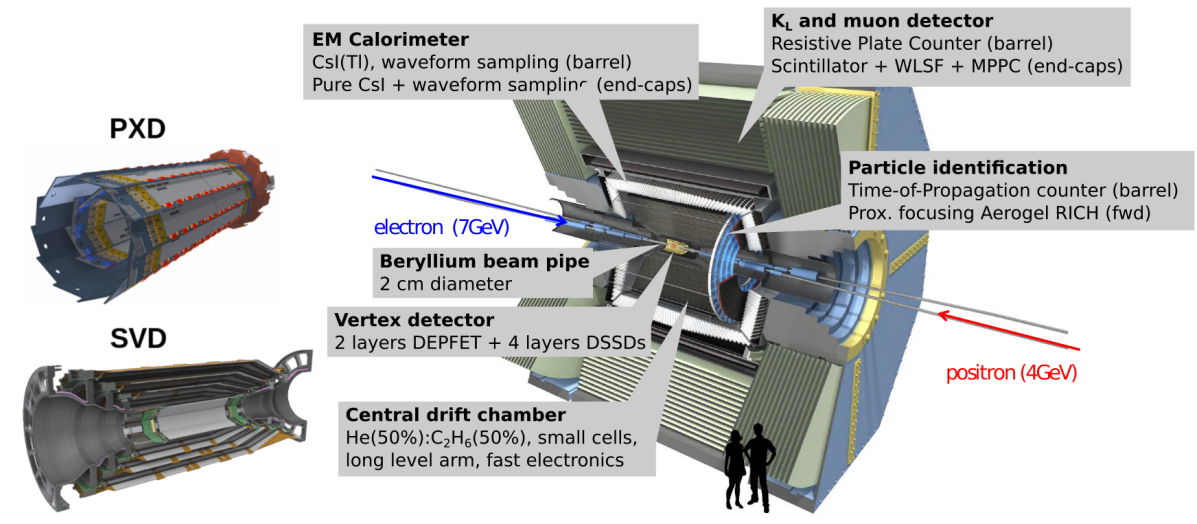

Figure 1: Cross-section of the Belle II detector with its sub-detector systems. Pixel (PXD) and strip (SVD) detectors are shown separately. Main new and/or upgraded features of each sub-detector are highlighted in the captions.

Table 1: The sub-detectors of the Belle II detector, their alignable elements and corresponding alignment parameters which are integrated in the common alignment procedure.

\begin{tabular}{l|l|l|c} 
sub-detector & alignable elements & alignment parameters & $\sum$ parameters \\
\hline IR & primary vertex position & $x, y, z$ & 3 \\
\hline VXD & 20 (PXD) + 187 (SVD) sensors & $u, v, w, \alpha, \beta, \gamma$ & $120+1,122$ \\
& & $P_{20}, P_{21}, P_{22}$ & $60+561$ \\
& & $P_{30}, P_{31}, P_{32}, P_{33}$ & $80+748$ \\
& 10 (PXD) + 45 (SVD) ladders & $u, v, w, \alpha, \beta, \gamma$ & $60+270$ \\
& 4 half-shells & $x, y, z, \alpha, \beta, \gamma$ & 24 \\
\hline CDC & 56 layers & $x, y, \phi, d x, d y, d \phi$ & 336 \\
& 14,336 wires & $x_{B}, y_{B}, x_{F}, y F$ & 57,344 \\
\hline KLM & 120 modules (barrel) & $u, v, \gamma(+w, \alpha, \beta)$ & $360(+360)$ \\
& 104 segments (end-caps) & $u, v, \gamma$ & 312 \\
\hline
\end{tabular}

The alignable elements and related alignment parameters of the sub-detectors discussed in this contribution are summarized in Table $1^{2}$.

The alignable elements of VXD - sensors, ladders and half-shells, as well as KLM modules and segments are treated as rigid bodies with up to six alignment parameters $(u, v, w, \alpha$, $\beta, \gamma)$ - three shifts in their local coordinate system and three rotations around the respective local axes. For KLM modules, only three of the six available rigid body parameters are currently used for alignment. For VXD half-shells and the position of the primary interaction vertex, instead of $(u, v, w)$ we denote the parameters as $(x, y, z)$ to emphasize that these parameters correspond to the actual global coordinates of the detector $(z$ coordinate along the beam axis).

\footnotetext{
${ }^{2}$ Note that the values are referring to the maximal number of parameters exercised in MC simulations - in reality not all sensors are installed for PXD (10 instead of 20) and not all CDC wires are operational (also one layer is disabled)
} 
In addition, to account for sensor deformations, the surface of the VXD sensors is parametrized using Legendre polynomials with parameters $P_{n i}$ denoting the amplitudes of the $n$-th order contributions.

The CDC is organized in 56 layers with six alignment parameters - two shifts and a rotation in $R-\phi$ plane common to both end-plates $(x, y, \phi)$ and two relative shifts and rotation at the forward end-plate with respect to the backward end-plate $(d x, d y, d \phi)$. The wires are aligned relative to the layers using two offsets at the backward end-plate $\left(x_{B}, y_{B}\right)$ and the forward end-plate $\left(x_{F}, y_{F}\right)$.

In total there are over 60 thousand parameters to be determined. However, the alignment of CDC wires is not subject to a regular alignment setup, due to a limited amount of available cosmic ray tracks collected with the magnets switched off. Therefore values determined in earlier data taking periods are currently used for CDC wires and the regular alignment setup determines about three thousand parameters.

\section{Alignment and calibration software at Belle II}

There are significant efforts at Belle II to automate the determination of the calibration constants as much as possible. For that purpose, a common calibration and alignment framework (CAF) [4] is developed, which is part of the Belle II simulation and analysis framework (basf2) [6]. The individual calibrations are separated into collector modules, aggregating the data collection from reconstructed events; and algorithms, that determine the actual calibration constants including the alignment parameters. These components are written in $\mathrm{C}++$, while the configurations and workflow management are steered using Python scripts and high-level tools for task automation, providing a convenient user interface. The automated calibration is executed at KEKCC (KEK Computing Center) using Apache Airflow which executes expert-defined scripts with configured calibrations in a fixed software release (various Atlassian platform collaborative tools and git version control system supported by DESY IT infrastructure are used by the Belle II Collaboration), handling also upload of the calibration constants in form of payloads and corresponding intervals of validity to a conditions database [5] used across basf2 for retrieval of various, possibly time-dependent, parameters organized in so called global tags.

The alignment procedure is integrated in $\mathrm{CAF}$ and largely profits from standard tools of basf2, using the same methods as standard Belle II reconstruction [7], from extrapolation in inhomogeneous magnetic field to track fitting and vertexing. For track fitting, the GENFIT toolkit [8] is used, which allows for generic treatment of various measurement types, extrapolation in detector material and integration of different track fitting methods. We integrated the General Broken Lines track refit (GBL) [9] into GENFIT, which allows proper treatment of multiple scattering effects in the track fit and is suitable for use with the Millepede II [10] solver.

The alignment constants are determined by minimization of track-to-hit residuals by Millepede II algorithm, which efficiently solves the linearized $\chi^{2}$ minimization problem by simultaneous fit to all alignment and track parameters, taking into account correlations among the measurements and thus reducing possible systematic effects (weak modes). The collector module allows various track samples to be used as alignment input: from cosmic rays (with or without magnetic field) or collision and beam background tracks, to vertex- and beam-constrained decays or two-body decays with an invariant mass constraint.

Samples containing variety of track topologies are required for the alignment procedure. Already the basic combination of cosmic rays (in magnetic field) and collision track yields sufficiently good results (without wire alignment) and the resulting residual misalignment is 
mainly related to deformation of CDC leading to slight global offsets of detector origin (at several micrometer level, worse in $z$ direction, where $\mathrm{CDC}$ resolution is poor).

Varying the combination of several MC data samples (including also cosmic rays without magnetic field or currently very statistically limited mass constrained $J / \Psi \rightarrow \mu^{+} \mu^{-}$), as well as attaching weights (for example to compensate for low statistics of $J / \Psi \rightarrow \mu^{+} \mu^{-}$), was studied on simulations. It is generally confirmed that adding more topologies and constraints reduces residual misalignment. However, for example the above mentioned $z$ bias in global offset is not reduced (in fact seems to grow by several $\mu \mathrm{m}$ by addition of $e^{+} e^{-} \rightarrow \mu^{+} \mu^{-}$). For the standard configuration (without wire alignment), no further data samples seem to be needed for an alignment which has negligible impact on physics performance. Studies on physics analyses using the residual misalignment from simulations are ongoing, but at the current integrated luminosity, many effects are not yet expected to be observable.

The potential of the additional samples is mainly seen in CDC wire alignment, where some constraints could be removed. For example, the radius of each CDC layer is currently fixed to nominal value. A radial expansion of $\mathrm{CDC}$ would result in a change of momenta of all particles. This degree of freedom can only be constrained by introducing an absolute mass scale - by adding mass-constrained decays with known invariant mass as an external measurement. Regarding the vertex- and beam-constrained $e^{+} e^{-} \rightarrow \mu^{+} \mu^{-}$, this sample seems essential for a good wire alignment, in addition to the basic samples mentioned above, and should be included in the standard procedure preferably (after additional studies).

Also the simultaneous determination of alignment constants of various sub-detectors is preferred, which would otherwise need to be treated by iterating among internal and relative alignments of the sub-detectors, making the estimation of possible systematic errors difficult and the procedure computationally expensive. This is exercised to various degree using simulations, with promising results. However further developments are needed, especially to run KLM alignment together with VXD and CDC and to handle time-dependence of IP position in data properly (to use IP-constrained di-muons). On the other hand, simultaneous alignment of CDC layers and VXD sensors with cosmic ray tracks recorded during operation combined with tracks from collision and beam background events is well understood and performed regularly.

The computing resources needed for regular alignment (in so called prompt calibration - spanning about two weeks of data-taking) of about three thousand parameters are completely dominated by the data collection, which is parallelized on a computing cluster and with about 500 jobs, a full reconstruction of $\sim 1 \mathrm{M}$ cosmic ray and $\sim 0.5 \mathrm{M}$ tracks from collisions takes about one hour. Typically two or three iterations with full reconstruction using updated constants are performed - until the parameter corrections are on average compatible with statistical errors. For this small problem (no wire alignment), we utilize the diagonalization method in Millepede II, taking only minutes to achieve the solution (and about 3 GB of memory). For the full configuration with alignment of wires, reaching 60 thousand parameters, a variant of generalized minimization of residuals is employed for solution. In this case, the running time of Millepede II for single iteration is $\sim 1 \mathrm{~h}$ and the required memory (namely to store the global matrix) is $\sim 19 \mathrm{~GB}$.

\section{First alignment and performance results}

Initially, CDC layers and VXD were aligned using cosmic ray data without magnetic field in 2019. The quality of the VXD alignment was verified and checked for presence of systematic deformations (radial expansion, bowing, telescope etc.) using the overlap residual method [11]. This method uses hits on tracks which cross a silicon layer twice in the overlap region 

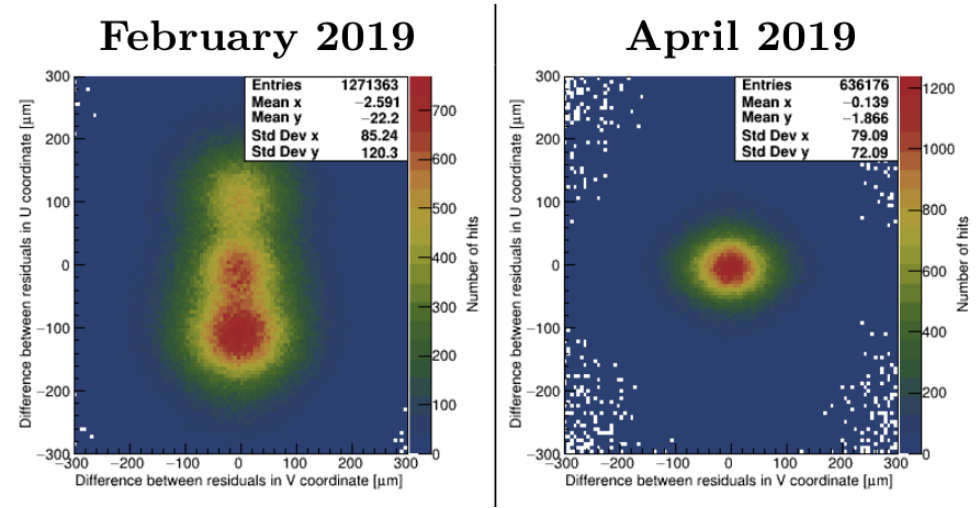

Figure 2: Overlap residual distributions from all VXD sensors in 2019 cosmic data before fixing SVD sensor pitch (left) and after (right).

of the sensors. It is a powerful tool to detect systematic distortions and can often unambiguously distinguish among deformations like radial expansion or twist deformation of the vertex detector. The disadvantage of this method is that rather large statistics needed because fewer than $10 \%$ of tracks have an overlap hit doublet.

While standard track-to-hit residuals did not show any significant alignment issues with the initial cosmic ray data in 2019 , the overlap residual method was able to detect an effect resembling radial expansion, when compared to MC simulation on misaligned detector - a snowman structure visible in Fig. 2 (left). We identified the cause of the issue as a slightly wrong pitch of the SVD sensors used in reconstruction ${ }^{3}$, which was compensated by the alignment procedure by changing the radii of the SVD layers. After fixing the sensors' pitch, subsequent validation did not show any visible systematic distortions with the cosmic ray data.

With first collision data, the alignment was performed using cosmic ray tracks in magnetic field and single tracks from collision and beam background events. The use of vertex and beam constrained di-muon events is postponed to higher luminosities due to limited amount of such events and the necessity to understand the time dependence of primary vertex position. Also the cosmic ray tracks without magnetic field were not used, due to suspected deformations induced when the solenoid magnet is excited. We shortly discuss how to deal with such issues in Section 5.

The overlap residual validation was repeated for collision data and confirmed the absence of significant systematic deformations of the vertex detector. We estimate any traditional weak mode amplitude must be smaller than about $30 \mu \mathrm{m}$, otherwise it would be visible with this method. According to simulation studies of the alignment, systematic deformations in VXD should be suppressed to better than $\sim 5 \mu \mathrm{m}$. Additional validations were possible with the collision data. As an example, we discuss here the evaluation of the transverse impact parameter $\left(d_{0}\right)$ resolution of the detector using two-track events from Bhabha and di-muon events.

To estimate the impact parameter resolution, a standard method is to compare the parameters of the two tracks with a common vertex at the point of closest approach to the origin

\footnotetext{
${ }^{3}$ The wrong value was in an XML file used for geometry definition - this was unnoticed because instead of pitch, the size of the active area and the number of strips were used as parameters in XML to calculate the pitch later. A slightly imprecise number was used for the size of the active area, resulting in a different pitch of the sensors.
} 

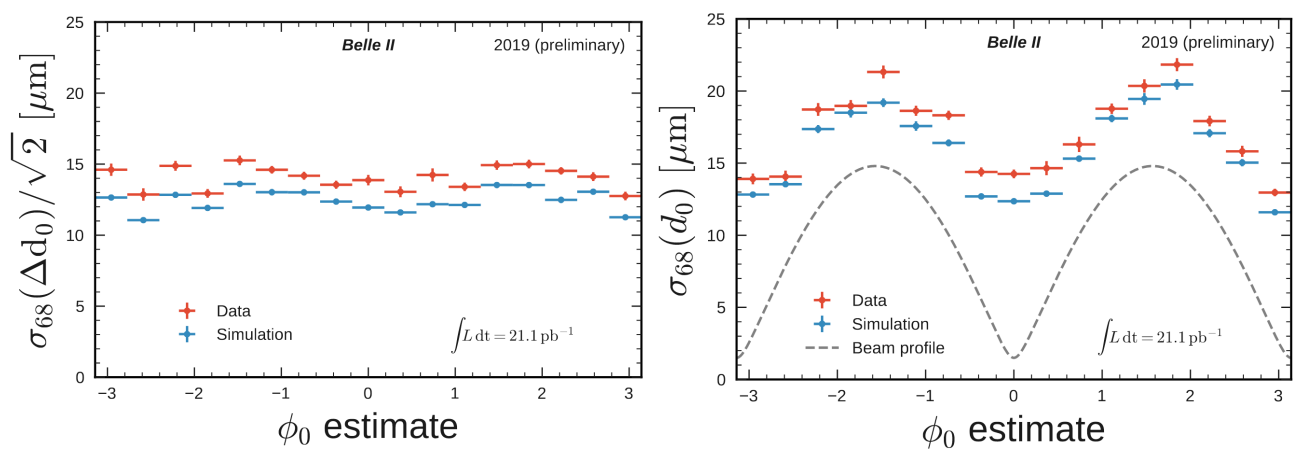

Figure 3: Transverse impact parameter resolution vs. $\phi_{0}$ of a (positive) track, for di-muon and Bhabha events collected in early 2019 data, estimated with the standard method (left) and the method utilizing the very small luminous region size in the vertical direction (right). The beam profile (dashed line, which corresponds to $\sigma_{i}=0 \mu \mathrm{m}$ ) and simulation (blue) is using horizontal and vertical size $\sigma_{x}=14.8 \mu \mathrm{m}$ and $\sigma_{y}=1.5 \mu \mathrm{m}$, respectively, calculated from machine parameters. Here $\sigma_{68}$ denotes half of the symmetric range about the median containing $68 \%$ of the distribution.

and plot their difference $\Delta d_{0}$. Another method, taking advantage of the very small size of luminous region in vertical direction of $\sigma_{y} \sim 1.5 \mu \mathrm{m}$ (which will get even smaller in future), allows an independent check. For nearly horizontal tracks, the variance of $d_{0}$ gives almost a direct estimate of the transverse impact parameter resolution. The dependence of the width of $d_{0}$ distribution on $\phi_{0}$, the angle of the track with respect to the vertical direction, is expected to follow $\sigma\left(d_{0}\right)=\sqrt{\sigma_{i}^{2}+\left(\sigma_{x} \sin \phi_{0}\right)^{2}+\left(\sigma_{y} \cos \phi_{0}\right)^{2}}$, where $\sigma_{i}$ is the actual detector resolution in the transverse impact parameter, which can be obtained from a fit. The comparison of these two methods is shown in Figure 3, including a comparison to the MC simulation. A transverse impact parameter resolution of $14.1 \pm 0.1 \mu \mathrm{m}$ is obtained consistently with both methods. The slight discrepancy to the simulation is seen consistently in both cases. This discrepancy is understood as an overly optimistic MC simulation for SVD sensors and is under further investigation.

\section{Summary and Plans}

The detector calibration and alignment are essential for precision physics. We developed a global alignment method using Millepede II for primary vertex position, pixel detector, strip detector, drift chamber and muon system and integrated it with common tools and workflows. The method was successfully exercised with simulations and first experiment data and subsequently validated with multiple methods. The status of the method presented in this contribution constitutes only initial results and is will be substantially extended with the coming data and increased luminosity of the accelerator.

The next steps will involve not only efforts in further automation of the procedure, but also advances resulting from better understanding of the detector and the data. The main concern is time dependence of the alignment constants. As an example, di-muon events with beam and vertex constraint are known to substantially improve the precision of the method, but as the primary vertex position is run dependent, it has to be aligned simultaneously with the detector components. This requires enough di-muon events in each run for its determination. 
The method of global simultaneous alignment allows the time dependence to be considered, too. For limited amount of the alignment constants describing alignment of larger structures (like VXD half-shells), more intervals of validity are considered, while for example sensor-by-sensor alignment is determined only once in the simultaneous fit. Similarly, if the deformation due to magnetic field can be described well with the current parametrization on the level of larger structures, cosmic ray data without magnetic field can be included in the alignment together with the collision data. This requires further studies and careful validations, also on a run-by-run basis and is foreseen with increased amount of available data.

\section{References}

[1] Z. Doležal, S. Uno et al., Belle II Technical Design Report, High Energy Accelerator Research Organization (2013) arXiv:1011.0352.

[2] C. Marinas et al. [DEPFET Collaboration], The Belle II pixel detector: High precision with low material, Nucl. Instrum. Meth. A 731 (2013) 31.

[3] K. Adamczyk et al., The silicon vertex detector of the Belle II experiment, Nucl. Instrum. Meth. A 824 (2016) 406.

[4] D. Dossett et al., Status of the calibration and alignment framework at the Belle II experiment J. Phys.: Conf. Ser. 898 (2017) 032032

[5] L. Wood et al., Conditions Database for the Belle II Experiment J. Phys.: Conf. Ser. 898 (2017) 042060

[6] Kuhr, T., Pulvermacher, C., Ritter, M. et al. The Belle II Core Software, Comput Softw Big Sci 3 (2019) 1

[7] T. Hauth (Belle II Tracking Group), Belle II Track Reconstruction and Results from first Collisions, EPJ Web Conf. 214 (2019) 02032.

[8] J. Rauch and T. Schlüter, GENFIT - a Generic Track-Fitting Toolkit, arXiv:1410.3698.

[9] C. Kleinwort, General Broken Lines as advanced track fitting method, Nucl. Instrum. Meth. A 673 (2012) 107.

[10] V. Blobel, C. Kleinwort, and F. Meier, Fast alignment of a complex tracking detector using advanced track models, Comp. Phys. Commun. 182 (2011) 1760.

[11] J. Kandra, T. Bilka et al., B lifetime and $B^{0}-\bar{B}^{0}$ mixing results from early Belle II data, arXiv:1906.08940 (2019) 